\title{
El Breve “Discurso del Método” de Claude Shannon
}

Claude SHANNON's BRIEF "Discourse ON METHOD”

\author{
JUAN RAMÓN ÁLVAREZ \\ Universidad de León, EsPAÑA \\ jralvarezb@gmail.com
}

\begin{abstract}
The following study departs from the lecture, entitled "Creative thinking", delivered by Claude Shannon in 1952 at the Bell Laboratories. This paper includes an interpretive and critical account of the necessary conditions (innate and acquired), as well as the desirable procedures, which must be satisfied in the scientific and technological invention, within the frame of the so-called scientist's spontaneous philosophy (SSP).
\end{abstract}

Keywords: Analogy $\bullet$ generalization $\bullet$ method $\bullet$ model $\bullet$ Shannon.

"Claude Shannon no fue simplemente una mente teórica brillante - fue también una mente extraordinariamente fértil, divertida, práctica e inventiva. Hay muchos matemáticos e ingenieros que escriben grandes trabajos. Hay menos que, como Shannon, sean también malabaristas, monociclistas, cachareadores, ajedrecistas de primera, descifradores de códigos, analistas financieros y poetas aficionados".

Goodman y Soni, 10,000 hours with Claude Shannon...

\section{Introducción}

La metodología de la ciencia, como teoría de los procedimientos simbólicos, técnicos y sociales en el marco de los diversos campos científicos, pugna por conservar tanto su existencia como su identidad. Como ya indicó a finales del siglo pasado Fernández Buey (1991), la cuestión de esa identidad está a horcajadas de una tendencia divisoria que segrega, por un lado, una metodología filosófica asimilada a una teoría general de la racionalidad científica — versión generalista — y, por el otro, del aprendizaje de técnicas sumamente especializadas por parte de los científicos — versión 
particularista. Tal brecha en expansión haría cada vez más difícil el intercambio de ideas entre filósofos generalistas, alejados de la práctica efectiva particular de las diferentes ciencias y científicos progresivamente convertidos en tecnólogos, apartados cada vez más de una teoría general de los procedimientos comunes a las diversas ciencias. Contra esta tendencia he insistido en que existe un territorio propio de la metodología de las ciencias que posee identidad propia respecto de esa comunidad (Álvarez 2011), aunque no tenga la pretensión de reducir a unidad sin fisuras esa pluralidad científica, la famosa desunión (disunity; Dupré 1983; 1993) de las ciencias, sostenida en España de forma mucho más exhaustiva por Bueno en su teoría gnoseológica del cierre categorial (Bueno 1992).

\section{El cruce de dos dicotomías}

En la introducción anterior están implícitas dos dicotomías, que hago ahora explícitas con el fin de ordenar el marco de la discusión metodológica. La primera de ellas opone una perspectiva filosófica a una perspectiva científica; la segunda, enfrenta una metodología unitaria a una pluralidad de procedimientos vinculados a la desunión científica. El resultado de combinarlas es la tabla siguiente:

\begin{tabular}{|c|c|c|}
\hline METODOLOGÍA & A. Unitaria & B. Pluralista \\
\hline 1. Científica & Naturalismo metodológico & Pluralismo tecnológico \\
\hline 2. Filosófica & Ciencia unificada & Desunión gnoseológica \\
\hline
\end{tabular}

Tabla 1

1A. El naturalismo metodológico se caracteriza, en tanto que naturalista, porque, acogiéndose a la tradición que considera ciencias fundamentalmente a las naturales, defiende que los procedimientos metodológicos son comunes a todas las ciencias y que se han ido extendiendo progresivamente a lo largo de la historia a las demás. En cuanto unitario, aunque lo es, no contrae el compromiso ontológico fuerte de reducir los objetos o procesos de las diferentes ciencias a los de las naturales y es, por tanto, compatible con la autonomía ontológica de las ciencias semióticas y humanas. ${ }^{1}$

1B. Llamo "pluralismo tecnológico" a la concepción científica que considera irreductibles entre sí las diferentes técnicas propias de cada ciencia atendiendo sobre todo a criterios de irreductibilidad ontológica. Si 1A es compatible con cualquier ontología, 1B excluye el reduccionismo ontológico propiamente dicho, sea a las ciencias fundamentales - física y química - o a las basales de un nivel $n$ respecto del nivel superior $n+1$, en cualesquiera pares de la jerarquía ontológica múltiplemente ordenada. 
2A. Considero ciencia unificada a la tesis metodológica de la filosofía de la ciencia que no unifica en vertical las ciencias por la dependencia de sus principios ontológicos, sino, por así decirlo, en horizontal por los procedimientos comunes a todas las ciencias. Descartes inauguró en la modernidad, frente al aristotelismo de las formas específicas, esta unificación bajo la idea de una mathesis universalis (Descartes 1965, p.360; Álvarez, 1988, pp.17-8), estructura común de todas las ciencias, en su concepción metodológica de la ciencia. Esta tradición llegó en el siglo XX hasta la teoría neopositivista de la ciencia unificada bajo criterios lingüísticos, estandarte de dicho movimiento.

2B La desunión gnoseológica, además de en Dupré (1983, 1993), en su versión más fuerte y también más completa, se sostiene en la teoría del cierre categorial de Gustavo Bueno (1992). En la misma, la perspectiva llamada gnoseológica, diferente tanto de la epistemología como de la metodología, se sostiene la tesis de que los procesos de cierre categorial a través de las operaciones materiales de los sujetos construyen la realidad científica misma, es decir, transforman la ontología de los términos del campo de cada ciencia. La heterogeneidad de las ciencias no es neutralizable y, por ello mismo, no cabe unificar metodológicamente las diferentes ciencias bajo una cobertura metodológica única. Puesto que - según esta teoría — las ciencias proceden de las técnicas, conservan la heterogeneidad de su origen y este punto de vista en filosofía corresponde bastante bien con el del pluralismo tecnológico en la ciencia.

Las oposiciones, por así decirlo, mayores se dan en las diagonales: naturalismo metodológico/desunión gnoseológica y ciencia unificada/pluralismo tecnológico, porque en ellas se oponen ciencia y filosofía, así como unidad y pluralidad. Estas son dobles, mientras que las otras (horizontales y verticales) son simples. ${ }^{2}$

En su conferencia de 1952, Shannon - como en general hacen los científicos, que practican el método (uno o múltiple) y ejercen de metodólogos solo cuando se tercia - no se sitúa en ninguna de las casillas de la tabla: habla, al parecer, por experiencia. Pero si hubiera que asignarle alguna, puesto que no hace mayores distinciones entre las ciencias y la tecnología que practica, la más conforme sería la primera, la del llamado naturalismo metodológico, aunque en su caso sería más apropiado hablar de "matematismo metodológico", pues son las matemáticas, y no las ciencias naturales, las que orientan sus reflexiones, que abarcan a la vez la ciencia y la tecnología — las matemáticas y la ingeniería.

\section{El "discurso"}

El texto base de la conferencia y la propia conferencia se articulan en dos bloques, que podrían considerarse, respectivamente, los requisitos y los procedimientos del pensamiento creativo. Singh (2017) proporciona un buen esquema y añade elementos a través otras fuentes indirectas. La forma general es la siguiente: 


\subsection{Introducción: la fertilidad científica}

En el comentario que dedican a este texto en su biografía de Shannon, Soni y Goodman (2017, Kindle edition, posición 3377) lo presentan de este modo:

Shannon dejó poco tras de sí en forma de memorias y lo más que se acercó a una autobiografía fue una charla pronunciada en un aula de los Laboratorios Bell ... Intencionadamente, la charla no revelaba nada de su historial ni de su vida privada, pero era el tipo de autobiografía que le importaba: una ventana hacia las operaciones de su cerebro. Bajo la apariencia de una conferencia sobre "el pensamiento creativo", resultó ser un atractivo manual breve de instrucciones sobre la forma de aparecerse del mundo a los ojos de un genio del nivel de Shannon.

Una autobiografía genérica - un oxímoron, sin duda - el testimonio de "un científico cualquiera", ${ }^{3}$ eso sí, como diría un unamuniano, escrita por un científico de "carne y hueso" con una biografía que los autores citados han escrito con maestría. Allí Shannon dio una vuelta de tuerca para presentar, en forma de requisitos y reglas, las bases de la fertilidad científica. En la introducción se parte, sin embargo, por oposición al gran discursivo del método, Descartes, de una desigualdad fundamental. Pongo a continuación, en doble columna, las afirmaciones de Descartes y Shannon.

\begin{tabular}{|c|c|}
\hline DESCARTES & SHANNON \\
\hline $\begin{array}{l}\text { El buen sentido es la cosa mejor re- } \\
\text { partida del mundo...la facultad de } \\
\text { juzgar bien y de distinguir lo verda- } \\
\text { dero de lo falso...es por naturaleza } \\
\text { igual en todos los hombres... No bas- } \\
\text { ta, pues, tener un buen ingenio, lo } \\
\text { principal es aplicarlo bien... Por mi } \\
\text { parte, nunca he presumido de que mi } \\
\text { ingenio fuese en algo más perfecto } \\
\text { que el de los demás... (AT, VI, pp.1- } \\
\text { 3) }\end{array}$ & $\begin{array}{l}\text { Un porcentaje muy pequeño de la po- } \\
\text { blación produce la mayor proporción } \\
\text { de ideas importantes... Hay personas } \\
\text { en las que, si se dispara una idea en } \\
\text { su cerebro, se sacará del mismo me- } \\
\text { dia idea. Hay otras personas que es- } \\
\text { tán más allá de este punto en el que } \\
\text { se producen dos ideas para cada idea } \\
\text { recibida. Son las personas que están } \\
\text { más allá del codo de la curva. No quie- } \\
\text { ro parecer ególatra, no pienso estar } \\
\text { más allá del codo de esta curva... (Cfr. } \\
\text { Álvarez } 2018 b[1-2])^{4}\end{array}$ \\
\hline
\end{tabular}

Tabla 2

Dos puntos de partida, una presunción en Descartes, una constatación figurada gráficamente (lo imaginamos entonces frente a la gráfica, hoy más bien frente a un Power Point en Shannon). El primero es presuntamente optimista, el segundo empíricamente realista, pero ambos "modestos" (verdadera o falsamente). Supuesta la 
capacidad común del buen sentido (bon esprit, bona mens), toda persona está dotada por la naturaleza para ir "acrecentando siempre gradualmente $\langle\mathrm{su}\rangle$ ciencia", para llegar "al verdadero conocimiento de aquello de que fuera capaz" (Descartes 1965, p.371; Cf. Álvarez 1988, p.17). El segundo, realista estadístico, reconoce que se parte de una desigualdad radical — le bon sens n'est pas la chose du monde la mieux partagée - articula su razonamiento en requisitos y reglas procedimentales, para hacer comprensible que, cuando ambos se satisfacen, se puede ir más allá del codo de la curva, donde están Newton y Einstein. Ambos se guardan muy bien de ponerse por encima del común, pero Shannon en esto se compromete más que Descartes y pasa, en la precisión de los requisitos o condiciones necesarias, a establecer tres. Así lo plantea:

¿Ahora bien, qué es lo que eleva a alguien hasta esta parte de la curva? ¿Cuáles son los requisitos básicos? Pienso que podríamos establecer tres cosas que son realmente necesarias para la investigación científica o para cualquier tipo de invención o matemáticas o física o algo en esa línea. Pienso que nadie puede avanzar sin ninguna de estas tres. (Cf. Álvarez 2018b [1-2]).

Tal como se formula, se requieren las tres características a la vez, conjuntivamente que, cómo se verá, son naturales y culturales (nature and nurture), constitución y educación. Incluso, para ser más claros, el orden no debería haber sido el que figura en el texto (ABC: formación; inteligencia; motivación). Lo mismo considero del tercer requisito: de poco vale estar formado, si no hay motivación o vocación. Me voy a permitir, por tanto, alterarlo en la forma que sigue.

\subsection{Requisitos de la fertilidad científica}

\subsubsection{B: Inteligencia o talento}

La primera condición necesaria (la segunda en el orden de Shannon) son dotes "naturales" sin las cuales las otras dos, la vocación y la formación, no pueden satisfacerse. Algo ingenuo parece, transcurridos tantos años, que Shannon cifre (nunca mejor dicho para el gran "descifrador") la naturaleza inteligente en el CI, sobre todo después del ya clásico libro de Gould (segunda edición1996 [1981]) The Mismeasure of Man (La falsa medida del hombre). Sea como fuere, el lugar argumentativo sigue siendo el mismo, existe "una cierta cantidad de inteligencia o talento" sin la cual la motivación y la formación son inútiles - o eso al menos pensaba Shannon. Sobre la naturaleza biológica de esas condiciones el texto es confuso, pues afirma que tener un CI por encima de la media "es una cuestión de ambiente; la inteligencia es cuestión de herencia" (Cf. Álvarez 2018b [2]). Una confusión tanto más sorprendente en quien hizo su tesis doctoral en materia biológica: el álgebra de la genética teórica (Cf. Álvarez 2018b, nota ii). Dejo la partida en tablas (para el ajedrecista Shannon) y me 
limito a señalar que concibió la inteligencia o el talento como una condición natural, biológica.

\subsubsection{C: Motivación (o vocación)}

La segunda condición necesaria, que Shannon no asocia explícitamente a la naturaleza biológica (en este caso psíquica), pero que sí parece estarlo implícitamente, es la motivación que, con ilustración jazzista incluida (Cf. Álvarez 2018b, nota iv), "se tiene o no se tiene" (innata y no adquirida: quod natura non dat salmantica non praestat). Pero la motivación se desglosa en tres aspectos o componentes: la curiosidad, la insatisfacción y el placer o fruición.

Como hacen bien en destacar Soni y Goodman (2017, posición 3388) esto es el colmo de la confusión y se limitó a remitir la motivación, en primer lugar, a la curiosidad:

No intento hacer en absoluto un análisis profundo, pero mi impresión es que un buen científico tiene una buena dosis de lo que podemos llamar curiosidad. No profundizaré más allá de este punto. El sujeto quiere saber las respuestas. Simplemente tiene curiosidad de cómo hacen tic tac las cosas y quiere conocer las respuestas a las preguntas; y si ve cosas, quiere plantear preguntas y quiere conocer las respuestas a aquellas. (Cf. Álvarez 2018b [3]).

Y no siendo suficientemente clara la curiosidad, hace una segunda remisión, esta vez a la noción, a mi juicio tan poco esclarecedora como la curiosidad, de insatisfacción constructiva, aunque Soni y Goodman (2017, posición 3377), siendo los mejores conocedores, encabecen todo el comentario a este trabajo con esa expresión. Con perdón de los biógrafos, las vaguedades de Shannon alcanzan aquí el cénit.

[E]xiste constantemente una ligera irritación cuando las cosas no parecen suficientemente correctas; y pienso que esta insatisfacción es en la actualidad una fuerza impulsora esencial para los buenos científicos. (Cf. Álvarez 2018b [3]).

Finalmente, la motivación tiene que ver con el disfrute anticipado del placer o la fruición concurrente con el descubrimiento, una especie de efecto "eureka" que sirve de premio al esfuerzo. "Si he estado intentando probar un teorema matemático durante una semana más o menos y finalmente encuentro la solución, saco un gran placer del ello". (Cf. Álvarez 2018b [3]). "Para Shannon nada podía sustituir 'el placer de ver los resultados netos"' (Soni y Goodman 2017, posición 3396).

Y ahora, en mi orden, cumplidas las condiciones "cuasinaturales" de la inteligencia y la motivación, se añade la condición cultural de la formación profesional: 


\subsubsection{A: Formación y experiencia.}

Esta, por obvia, la había despachado al comienzo sin comentario. Supuesto que se cumplan las condiciones necesarias, la aspirada suficiente tendrá que alcanzarse a mayores. Así lo planteó:

[S] upuesto que una persona tenga estas tres propiedades en la medida suficiente para ser útiles: ¿existen algunos trucos, algunos artilugios que pueda aplicar al pensamiento, que puedan ayudar realmente en el trabajo creativo, en la obtención de las respuestas en el trabajo de investigación, en general, a hallar las respuestas a los problemas? (Cf. Álvarez 2018b [4]).

No obstante, como se verá en 3.3.3, la formación no es solo positiva, también puede ser negativa cuando contribuye a la fijación de obstáculos epistemológicos (Bachelard 1960) que obran en contra del planteamiento adecuado de los problemas. Esto que Shannon da por sentado sin mayores problemas, se le cuela - por decirlo así -, en el cuarto procedimiento, el cambio de perspectiva, incluso de lenguaje, en la investigación científica. A veces es necesario desprenderse de "rutinas de pensamiento" bien asentadas en las tradiciones de investigación (Laudan, 1977, p.81) $)^{5}$ e, incluso, aquellos que están menos experimentados, que están "verdes", obtienen mejores resultados (Cf. Álvarez 2018b [6]).

\subsection{Procedimientos (estrategias) de la investigación científica}

Shannon trata con poca solemnidad los procedimientos que recomienda. No les llama "reglas", como imperativos a seguir; los llama trucos (tricks) o artilugios (gimmicks). Son procedimientos, al parecer muy naturales, puesto que "los buenos investigadores los aplican inconscientemente; es decir, lo hacen automáticamente" (Cf. Álvarez 2018b [4]) y será una gran ventaja traerlos a la consciencia, pues hacerlo contribuirá a obtener mejores y más rápidos resultados en la investigación. Cuando son plenamente conscientes - como lo son para Shannon cuando los presenta - es posible considerarlos, como hacen Soni y Goodman (2017, posición 3399), estrategias. A continuación se tratan las seis que figuran en el texto y que pueden llamarse: simplificación, analogía, replanteamiento, generalización, análisis estructural e inversión del problema. Son seis procedimientos utilizables - conjunta o separadamente en diferentes situaciones. No se desprende del texto de Shannon que sean seis pasos que haya que seguir sucesivamente en cada problema planteado, como interpretan Goodman y Soni (2017b): "un proceso de seis pasos para resolver cualquier problema". 


\subsubsection{La simplificación}

Se trata de un procedimiento consistente en despejar los problemas planteados, que suelen estar "entremezclados con toda clase de datos extraños de una u otra especie", eliminando del problema "todo lo que no sea esencial; es decir, reducirlo al tamaño adecuado" (Álvarez 2018b [5]). Esta eliminación, sin embargo, debe contenerse dentro de límites adecuados, so pena de eliminar el propio problema de partida. El texto no es suficientemente claro a este respecto y no establece criterios de esa limitación. Aun así, es posible hacerse cargo de este procedimiento de tipo general, pues es utilizable en "no importa qué clase de problema - diseñar una máquina, desarrollar una teoría física o probar un teorema matemático o algo semejante" - (Álvarez 2018b [5]), relacionándolo con otras ideas afines como son la de idealización y la de minimización.

En su teoría de los modelos, Bunge (1975, p.40) llama "objeto modelo" al tipo ideal o esquema simplificado del que parte una teoría.

[P]uede darse el nombre de objeto modelo a cualquier representación esquemática de un objeto. Si el objeto representado es concreto, entonces su modelo es una idealización del mismo...[L]a representación es siempre parcial y más o menos convencional.

Weisberg (2007), tras caracterizar la acción de idealizar como la distorsión de la representación de un objeto o un conjunto de fenómenos, ha distinguido tres de clases de idealización, a las que llama idealización galileana, minimización e idealización de modelos múltiples. Me parece que a la simplificación de Shannon le corresponde la minimización.

La idealización minimalista es la práctica de construir y estudiar modelos teóricos que incluyen solamente los factores causales que dan lugar al fenómeno. Tal representación se llama muchas veces modelo mínimo del fenómeno. Dicho de forma más explícita, un modelo minimalista incluye solo aquellos factores que marcan una diferencia para la producción o el carácter esencial del fenómeno en cuestión. (Weisberg 2007, pre-print, p.5)

Sin limitar el contenido del modelo a sus componentes causales, y tomando literalmente las afirmaciones de Shannon, se pueden establecer los siguientes rasgos del modelo resultante de la simplificación. 1) Se eliminan los elementos presumiblemente accidentales ("datos extraños”) del contenido empírico presente, 2) se alcanza una forma "elemental" del problema que solamente contiene elementos esenciales ( $\sin$ los cuales no sería el que es), 3) se busca la solución al problema simplificado, a sabiendas de que no es la solución definitiva, para cuya obtención es necesario "añadir refinamientos a la solución de este hasta regresar a la solución de aquel de que se partía" (Álvarez 2018b [5]). 
Aquí solo existe un riesgo, el de desbordar, por así decirlo, las fronteras del nivel de resolución del planteamiento del problema y de los sistemas implicados en él. En ese caso, la recomposición por medio de los refinamientos sería imposible. La simplificación debe ser, por ello, importante, pero no excesiva. Con estas precisiones basta para el propósito que aquí me ocupa. Es presumible que el ejemplo más oportuno de simplificación es el conocido modelo de Shannon para el problema de la trasmisión de información en sistemas de comunicación de canales con ruido, cuya ilustración es la que sigue:

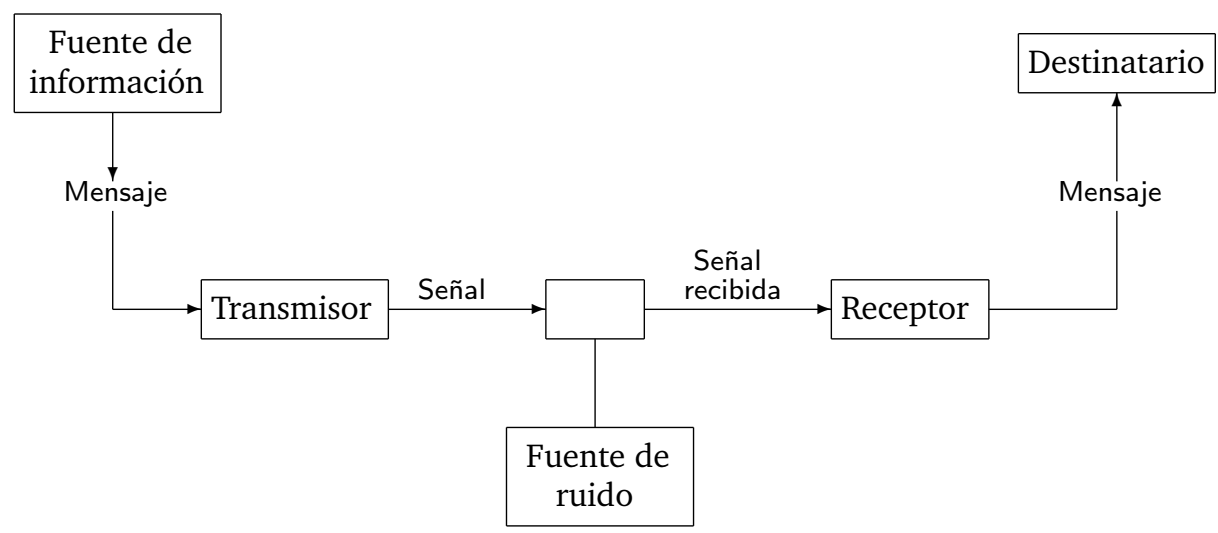

Figura 1

Así comentan al respecto Chiu et. al. ( 2001, p.19):

En este diagrama, que es la piedra de bóveda de su teoría de la comunicación, Shannon abstrajo ${ }^{6}$ (abstracted away) la mayoría de las complicaciones de los sistemas de comunicación. De hecho, cada parte del sistema es una simple caja.

Una vez hecha la simplificación fundamental, una vez establecida la morfología básica, se puede aplicar el instrumental matemático: la lógica booleana en la tesis de Máster sobre circuitos de interruptores, el álgebra en su trabajo en genética y la probabilidad en el caso de la comunicación.

\subsubsection{La solución analógica}

El segundo procedimiento está concisa y claramente formulado en el texto: la obtención de soluciones mediante analogías. Tal como lo presenta Shannon, se trata de la relación analógica de proporcionalidad existente entre cuatro términos: dos problemas y dos soluciones, cuando está planteado el problema cuya solución se desconoce 
y se conoce, sin embargo, otro problema semejante cuya solución es también conocida. Sea un problema en un campo $C$ en que somos expertos y donde se plantea un problema $P$, cuya solución $S$ se busca, en el cual conocemos tanto un problema $P^{\prime}$; semejante a $P$, como su solución $S^{\prime}$. Es decir, $P: S:: P^{\prime}: S^{\prime}$. Consiste este procedimiento en la determinación de un cuarto término, conocidos los otros tres. Leibiniz formuló esta relación, en la que se basa y define la relación de modelo (Álvarez 2018), con el nombre de "relación de expresión":

Se dice que expresa una cosa aquello en que hay relaciones (habitudines) que responden a las relaciones de la cosa que va a expresarse ... [P]or la contemplación de las relaciones de aquello que expresa podemos llegar al conocimiento de las propiedades que corresponden a la cosa que va a expresarse. Leibiniz (1678 pp.263-4; traducción, pp.178-9).7

Según la afortunada expresión de Esquisabel (2008, p. 14), rige aquí un principio de transferencia, "mediante el cual se puede pasar válidamente de las proposiciones verdaderas de uno a las correspondientes proposiciones del otro sin pérdida del rigor de la fundamentación". Si se tiene en cuenta que la relación de analogía de proporcionalidad es una relación de relaciones (las razones $A: B$ y $B: C$ ), se puede entender la afirmación final de Shannon, que caracteriza la ventaja metodológica del procedimiento: "[p]arece mucho más fácil hacer dos saltos pequeños que uno grande en cualquier clase de pensamiento mental" (Álvarez 2018b [6]). Esta es, diríase, la representación; la historia personal de Shannon proporciona de forma eminente el ejercicio analógico, en su tesis de máster de 1940 en el MIT: Análisis simbólico de los circuitos de relés e interruptores, cuyo adelanto fue el trabajo, del mismo título, de 1938 (Shannon 1993a, pp.471-95). En dicho texto se unen la lógica algebraica de Boole y los circuitos de interruptores en las inversas relaciones semánticas de representación e incorporación: ${ }^{8}$ el formalismo representa las redes corpóreas y las redes incorporan los símbolos.

Shannon resuelve analógicamente el problema ingenieril de la construcción de circuitos de interruptores (incorporaciones eficaces) mediante la analogía con los algoritmos (efectivos) del simbolismo booleano que da la solución.

Dadas ciertas características, se pide encontrar un circuito que incorpore estas características. La solución de este tipo de problema no es única... También se describirán métodos para hallar cualquier número de circuitos equivalentes a un circuito dado respecto de todas las características operativas (Shannon 1993a, p.471; cursiva añadida).

El problema $P$ es el diseño de un circuito, $S$ el diseño más eficaz; $P^{\prime}$ es la característica de una formula lógica compleja; $S^{\prime}$ la efectiva fórmula reducida, por ejemplo: "descubrió la forma de hacer el trabajo de once conexiones con sólo dos, una serie y un paralelo. Y esto sin tocar un solo interruptor" (Soni y Goodman 2017, posición 
747). Bien es verdad que hay dos campos diferentes — el de las máquinas eléctricas y el de los algoritmos lógicos - y no uno como en el planteamiento de la conferencia de Shannon. Hizo falta que Shannon fuera "experto" en lógica y en ingeniería eléctrica, para que la misma mente transitara de un dominio al otro.

\subsubsection{Los replanteamientos del problema: eliminación de los obstáculos epistemológicos}

En este punto Shannon se pronuncia de la forma más confusa sobre un procedimiento que debería estar claro, pero que se queda corta. Se limita a afirmar que se trata de

intentar replantearlo en tantas formas diferentes como sea posible... Si no se hace, es muy fácil caer en las rutinas del pensamiento mental... 〈sin lo cual) no nos podríamos liberar de determinados bloqueos mentales que nos mantienen en ciertos modos de contemplar un problema (Álvarez 2018b $[6])$.

Más que de una regla positiva, se trata de una regla negativa en el sentido de remover los obstáculos que impiden resolver el problema porque impiden plantearlo bien. Aquí hay dos referentes históricos a través de los cuales se puede entender a Shannon, a saber, los ídolos de Bacon y la noción de obstáculo epistemológico de Bachelard. Están los obstáculos de la comunicación y la tradición: en términos baconianos (Cf. Lafuente 1986, p.447) hay que cambiar las palabras, el lenguaje acostumbrado (idola fori) y las rutinas de pensamiento en que estamos instalados (idola theatri). El libro de Bachelard La formación del espíritu científico (Bachelard 1960) es en su integridad el desarrollo de dicha noción en sus diferentes etapas y variantes: diez obstáculos epistemológicos que van desde la experiencia primera hasta la sobrevaloración del conocimiento cuantitativo, considerado como libre de error.

\subsubsection{La generalización}

Shannon indica en este punto que la generalización es un procedimiento especialmente útil en las matemáticas. Sin duda, está pensando más en procedimientos de construcción conceptual que en la proyección ampliativa de la inducción como generalización de regularidades observadas.

El teorema de Pitágoras prueba que la suma del área de los cuadrados construidos sobre un triángulo rectángulo es igual al área del construido sobre la hipotenusa. Una generalización del mismo es su extensión de las áreas de los cuadrados a las de cualesquiera figuras semejantes. Otra generalización es desligarlo del triángulo rectángulo, cosa que ya conoció Euclides, pero que en su forma trigonométrica se conoce como ley del coseno o fórmula de Al-Kashi. Otras generalizaciones amplían la 
ontología de partida. Una cuadrática perfecta como $x^{2}-2 x+1=0$ puede resolverse como el producto $(x-1)(x-1)=0$, tal que $x=1$ satisface la ecuación. Es conocido el procedimiento de establecer la forma general $a x^{2}+b x+c=0(a \neq 0) \mathrm{y}$, por analogía con las cuadráticas perfectas, se procede a "completar el cuadrado" para hallar la forma general de solución. Sabido es que se consigue la fórmula general:

$$
x=\frac{-b \pm \sqrt{b^{2}-4 a c}}{2 a}
$$

de forma que si el discriminante $b^{2}-4 a c \geq 0$, entonces $x$ es número real; si $b^{2}-4 a c<$ 0 , entonces $x$ es un número complejo $a+b i$. No solo se ha generalizado la solución, sino que las soluciones son elementos del conjunto de los números complejos, con lo cual se ha ampliado la ontología merced a la metodología y según el principio de que toda operación debe tener resultado, es decir, ha de ser efectiva. Como en el caso del 0 , elemento neutro de la suma, el conjunto vacío - la intersección de dos conjuntos disyuntos - , etc., son las operaciones las que prevalecen, incluso en la escritura figurada de $\frac{1}{x}=\infty$, para $x=0$, donde ' $\infty$ ' no denota nada, simplemente indica que no hay valor para la función $f(x)=\frac{1}{x}$, cuando $x=0 ; f(0)=\infty$ : el colmo de un algebrista.

\subsubsection{Análisis estructural del problema}

Al presentar este procedimiento Shannon ofrece a la vez el concepto y el ejemplo, tomado, como no puede extrañar, de la lógica (axiomas y teoremas; premisas y conclusiones) Se trata de analizar, de descomponer, un largo camino en trayectos más pequeños, la estructura total en subestructuras o módulos. Esto vale tanto para el análisis estructural del software como del hardware, dada la semántica - representativa e incorporativa - en que se basa el constructo llamado "ordenador digital". Las derivaciones se componen de módulos (subderivaciones ligadas a las reglas elementales) y los circuitos lógicos se componen de módulos (circuitos elementales: series, paralelos, complementarios). 


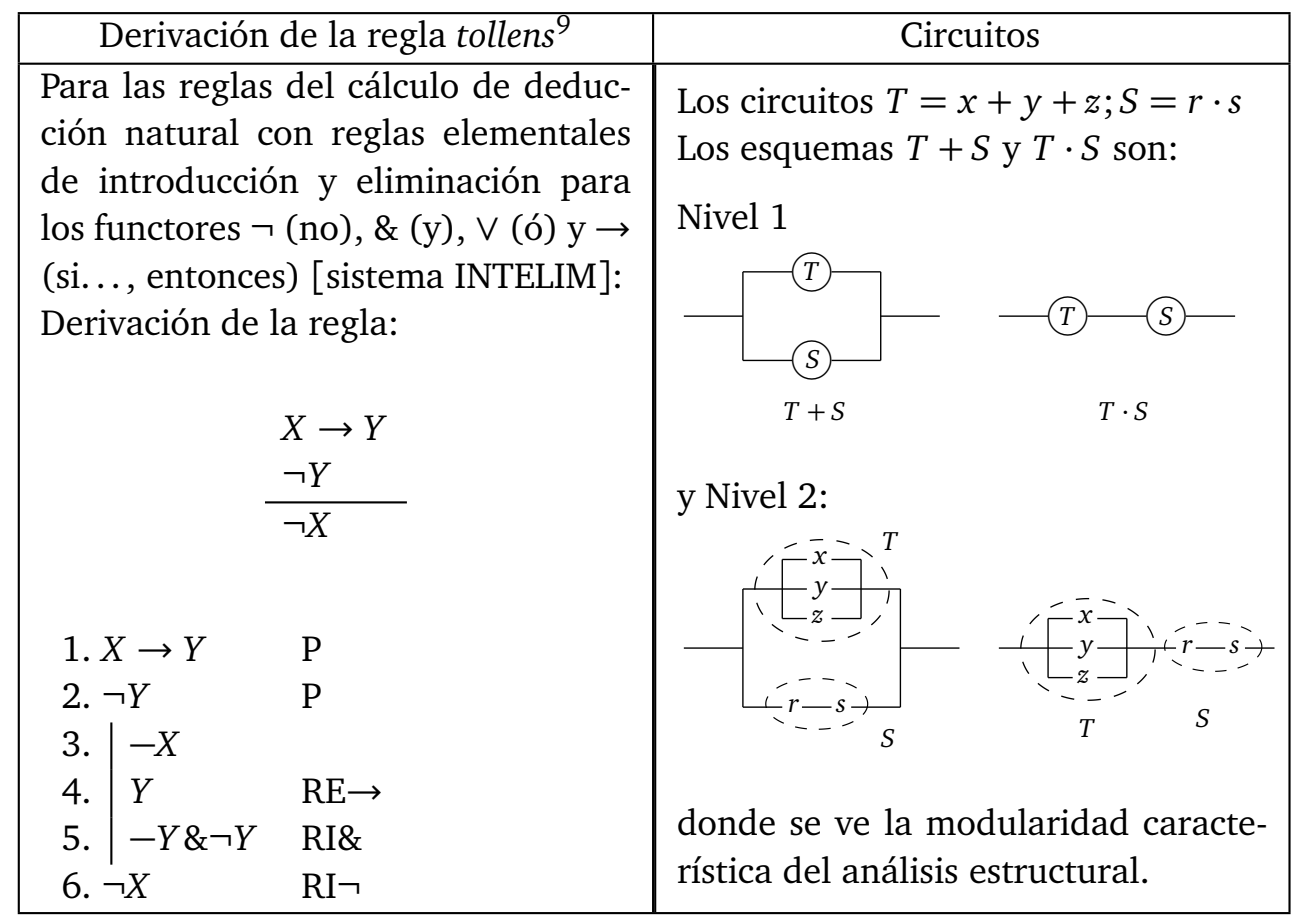

Tabla 3

\subsubsection{Inversión del problema}

Este procedimiento es, de los seis propuestos, el menos determinado — al menos me lo parece - aunque su presentación parezca muy sencilla: el intercambio de premisas y conclusión, de los datos y los resultados, y del diseño y el artefacto en cada caso. Basta con este primer desglose para hacer constar su intención y dificultades.

En el caso de los esquemas de argumento o de reglas de los mismos la inversión no plantea problemas si premisas y conclusiones son formalmente intercambiables, es decir, cuando

$$
\frac{p \rightarrow q}{\neg q \rightarrow \neg p} \quad \text { ó } \quad \frac{X \rightarrow Y}{\neg Y \rightarrow \neg X}
$$

(inversión salva consequentia), pero no cuando la inversión altera la consecuencia, caso en el cual la marcha atrás (ahora hacia adelante) de conclusión a premisas no se sigue, sino que se busca y se pasa de una deducción a una abducción: su versión mecanicista es la inversión de causa y efecto.

En el caso de los datos a los resultados, se puede volver a plantear el procedimiento directo y el inverso entre la multiplicidad de partida y su versión reducida. 
Wilkins y Ebach (2014) han argumentado que la clasificación es un procedimiento preteórico, consistente en el reconocimiento de patrones, diferente de la inducción y la deducción (Wilkins 2013), por el cual se reduciría la dispersión de los datos. También, hoy en día la utilización de potentes programas en los enormes acúmulos propios de los big-data, proporcionan una versión de alta tecnología que cumple función semejante a los mecanismos cognitivos de la clasificación.

En el caso de la inversión de diseño y artefacto se opondría la ingeniería directa (forward) a la inversa (reverse), tema muy tratado sobre todo en el mundo de la informática, en la búsqueda de los códigos fuente de determinados programas protegidos por patentes. Pero que, por razones de actualidad, el ejemplo del software sea el más conocido no hace menos cierto que esta inversión se utilice en campos diversos de la tecnología desde la aeronáutica hasta la arqueología (Cf. Neamtu et al., 2012).

Para tratar con justicia estos comentarios de Shannon debe tenerse en cuenta el tiempo transcurrido desde 1952 y los cambios descomunales que han tenido lugar en la ciencia y la tecnología desde entonces. No obstante, en las tres variantes anteriores de la inversión metodológica, se vuelve a encontrar el problema que Kant planteó en la Crítica de Juicio en relación con el juicio teleológico. Frente al juicio determinante, propio de la ciencia de la naturaleza (Mecánica), que subsume los casos en lo universal dados ambos los casos y la ley, cuando solo están dados los casos, cuando solo lo particular está dado, entonces ha de emprenderse la búsqueda de lo universal siguiendo un imperativo heurístico en una reflexión orientadora (juicio reflexionante) que, en el caso de los organismos, encontraba en la idea regulativa de finalidad. (Cf. Álvarez 1998). Esto nos sitúa en una tradición filosófica que nos lleva fuera del planteamiento literal de Shannon. Hay que volver, por tanto, a él, para encontrar las relaciones entre metodología científica y filosófica.

\section{La filosofía espontánea de los científicos (FEC) y el naturalismo metodológico}

En la introducción a su Curso de filosofía para científicos, Althusser (1975, pp.99-177) acuñó la expresión "filosofía espontánea de los científicos" para caracterizar "únicamente de las ideas ("conscientes", o no) que los científicos se hacen de la practica científica de las ciencias y "la" ciencia" (Althusser 1975, p.101). Distinta de las concepciones del mundo de los propios científicos, la FEC tiene un elemento interno, intracientífico, que consta, a su vez, de tres creencias: 1) en un mundo exterior real, 2) en la objetividad del conocimiento científico y 3) en "la corrección y la eficacia de los métodos de la experimentación científica, o método científico" (Althusser 1975, p.101). En España, Bueno asumió la expresión, también de forma negativa como variante de la filosofía inmersa o adjetiva (Bueno 1995, p.39) que practican tanto 
científicos como algunos filósofos sobre todo positivistas o neopositivistas. Tomaré aquí la expresión, no en el sentido negativo de Althusser o Bueno, sino en el de las consideraciones que, basadas en la experiencia de una práctica científica fructífera, manifiestan destacados científicos, como aquí Shannon, ante públicos de colegas como contribución ilustrativa. Me limitaré a la tercera creencia de Althusser, aunque extendida no sólo a la ciencia experimental, sino a todas las formas de ciencia, que en el caso de Shannon toman la forma de ciencias matemáticas cuya "experimentación" es el diseño y la validación de los ingenios producidos.

Así entendida, esta FEC se acerca a la versión o parte científica de la metodología de las ciencias que, junto a su versión filosófica, constituyen ambas la metodología general de la(s) ciencia(s), entre los extremos señalados al principio: una metodología filosófica excesivamente generalista (teorías de la racionalidad) y una(s) científica(s) excesivamente particularistas (aprendizaje y desempeño de técnicas especializadas). En primer lugar, con la referencia a su experiencia personal Shannon no se refiere a técnicas especializadas, sino a procedimientos genéricos intermedios incorporados materialmente a la investigación y el diseño; en segundo lugar, lo hace reflexionando sobre ellos sin poner más ejemplos que los de su quehacer personal. A su reflexión espontánea de científico virtuoso le falta lo que incluso a veces le sobra a la metodología filosófica de la ciencia, perspectiva y cultura histórica suficientes, mientras que posee lo que a esta última le falta, concreción y experiencia. Con la fácil paráfrasis que se suele hacer de una conocida expresión de Kant, podría decirse que la metodología científica sin la perspectiva filosófica es ciega y la filosófica sin la experiencia científica es vacía.

Esta visión espontánea, en su dimensión metodológica, se aproxima a lo que en la sección 2 se llamó naturalismo metodológico o, si se quiere apurar más el parecido, coincide en el límite con él. Podría decirse, pues, que la metodología espontánea de los científicos, aquí ejemplificada por el breve discurso de Shannon, es el naturalismo metodológico.

\section{Conclusión}

El escrito, poco elaborado por cierto, que sirvió de base a la conferencia de 1952, contiene consideraciones, apoyadas en su autoridad científica personal, que se pronuncian sobre los requisitos innatos y adquiridos de los investigadores científicos e inventores tecnológicos, así como sobre la naturaleza y las características de los procedimientos que son comunes a las diversas ciencias - e incluso a las tecnologías indisociables de algunas de ellas. Estos requisitos y procedimientos se sitúan, sin mayores justificaciones, en la franja que le corresponde a una metodología general de las ciencias que, tanto reflexivamente científica (no hay ciencia puramente refleja) como 
filosóficamente provista de semántica (la metodología general no es abstracta, está llena de historia), se aclaran y comentan en el texto que antecede como producto de la filosofía espontánea de este científico creador. Cobijada bajo esta denominación, tomada aquí en un sentido positivo, representa una dimensión esencial de la ciencia realmente existente y como posición metodológica es el naturalismo metodológico. Cumplidos setenta años de la publicación de "Una teoría matemática de la comunicación", y sesenta y cinco de "Pensamiento creativo", este breve discurso sigue siendo buena ocasión para tratar un tema recurrente en la historia de la ciencia.

\section{Referencias}

Althusser, L. 1975[1967]. Curso de filosofía para científicos. Introducción: Filosofía y filosofía espontánea de los científicos. Traducción de A. Roies. Barcelona: Laia.

Álvarez, J. R. 1988. Ensayos metodológicos. León: Universidad de León.

. 1998. Una débil esperanza: la idea kantiana de una ciencia biológica. In: J. Arana

(ed.) Los filósofos y la Biología, pp.49-66. Sevilla: Universidad de Sevilla.

- 2011. Metodología de la ciencia: Tratados, manuales y textos. In: R. Bestani et al. (Comp.) Textos, autores y bibliotecas, p.453-67. Córdoba: Universidad Nacional de Córdoba.

- 2018a. Modelos y analogías: formas de la expresión. Scripta Philosophiæ Naturalis 14: $37-63$.

- 2018b. Traducción de Creative Thinking de Claude Shannon. In: https: //www.academia.edu/37359081/Traducci\%C3\%B3n_de_Claude_Shannon_Creative Thinking_Pensamiento_creativo Consultado 09/09/2018.

Bachelard, G. 1960. La formation de l'esprit scientifique. Contribution a une psychanalyse de la connaisance objective. $4^{a}$ edición. Paris: J. Vrin.

Bueno, G. 1992. Teoría del cierre categorial. Oviedo: Pentalfa, 5 vols.

- 1995. ¿Qué es la filosofía? $2^{a}$ edición. Oviedo: Pentalfa.

Bunge, M. 1975. Teoría y realidad. Traducción de J. L. García Molina y J. Sempere. $2^{a}$ edición. Barcelona: Ariel.

Chiu, E. et al. (eds.) 2001. Mathematical Theory of Claude Shannon. A study of the style and context of his work up to the genesis of information theory. http://web.mit.edu/6.933/www/ Fall2001/Shannon1.pdf Consultado 15/04/2018.

Descartes, R. 1964-1978. Oeuvres de Descartes. Edición de Ch. Adam y P. Tannery, nouvelle présentation mise à jour par B. Rochot. París: CNRS-J. Vrin, 11 vols. (Citadas como AT + volumen + número de página).

— 1965. Regulae ad directionem ingenii. Edición de H. Gouhier. $4^{a}$ ed., París: J. Vrin.

Dupré, J. 1983. The disunity of science. Mind 92(367): 321-46.

- 1993. The Disorder of Things: Metaphysical Foundations of the Disunity of Science. Massachusetts/London: Harvard University Press.

Esquisabel, O. M. 2008. Leibniz y el concepto de analogía. Revista de Filosofía y Teoría Política 39: 11-29. 
Fernández Buey, F. 1991. La ilusión del método. Ideas para un racionalismo bien temperado. Barcelona, Crítica.

Goodman, R.; Soni, J. 2017a. 10,000 Hours with Claude Shannon: How a Genius Thinks, Works, and Lives. https://medium.com/the-mission/10-000-hours-with-claude- $\mid$ shannon-12-lessons-on-life-and-learning-from-a-genius-e8b9297bee8f $16 / 04 / 2018$.

- 2017b. One of the fathers of modern computing used this 6-step process to solve any problem. http://www.businessinsider.com/engineer-claude-shannon- $\backslash$ problem-solving-process-2017-7> Consultado 10/05/2018.

Gould, S. J. 1996[1981]. The Mismeasure of Man. $2^{a}$ edición. New York: W.W. Norton.

Lafuente, $\mathrm{M}^{a}$ I. 1986. La reforma filosófica de Francis Bacon. In: S. Onega (ed.) Estudios literarios ingleses. Renacimiento y Barroco, pp.437-66. Madrid: Cátedra.

Laudan, L. 1977. Progress and its Problems. Towards a Theory of Scientific Growth. Berkeley/Los Angeles: University of California Press.

Leibniz, G. W. 1678. Quid sit idea. In: C. J. Gerhardt, G. W. Leibniz, Die philosophischen Schriften, pp.263-4. Hildesheim: Georg Olms, Vol. VII. (Traducción española en Leibniz, G. W. Escritos filosóficos. 1982. Edición de Ezequiel de Olaso. Buenos Aires: Charcas.)

Neamtu, C. et al. 2012. Using Reverse Engineering In Archaeology: Ceramic Pottery Reconstruction. Journal of Automation, Mobile Robotics \& Intelligent Systems 6(2): 55-9.

Shannon, C. E. 1948. A Mathematical Theory of Communication. Reprinted with corrections from The Bell System Technical Journal, Vol. 27, pp.379-423, 623-656, July, October, 1948. http://math.harvard.edu/ ctm/home/text/others/shannon/entropy/entropy.pdf Consultado 15/04/2018.

- 1952. Creative Thinking. Texto establecido por Jimmy Soni. https://medium. com/the-mission/a-genius-explains-how-to-be-creative-claude-shannons-long-lost-1952- $\backslash$ speech-fbbcb2ebe07f Consultado 14/04/2018.

- 1993a. Collected Papers. Edited by N. J. Sloane and A. D. Wyner. New York: IEEE.

- 1993b. Miscellaneous Writings. Edited by N. J. A. Sloane and A. D. Wyner. Scanner Internet Archive HTML5 Uploader 1.4.1. https://archive.org/details/ ShannonMiscellaneousWritings. Consultado 15/04/2018.

Singh, J. T. 2017. Claude Shannon's 1952 Lecture on CreativeThinking. https://spinlab.me/ 2017/07/30/claude-shannons-1952-lecture-on-creative-thinking Consultado 15/04/2018.

Soni, J. 2017. Claude Shannon's “Creative Thinking” Speech: A Genius Reveals How To Be Creative. https://medium.com/the-mission/a-genius-explains-how-to-be-creative- $\backslash$ claude-shannons-long-lost-1952-speech-fbbcb2ebe07f Consultado, 19/04/2018.

Soni, J.; Goodman, B. 2017. A Mind at Play. How Claude Shannon Invented the Information Age. (Kindle edition). New York: Simon \& Schuster.

Weisberg, M. 2007. Three kinds of idealization. The Journal of Philosophy 104(12) 63959. Versión pre-print del autor. https://repository.upenn.edu/cgi/viewcontent.cgi?article= $1013 \&$ context=philosophy_papers Consultado 25/04/2018.

Wilkins, J. S. 2013. Pattern recognition: neither deduction nor induction. http://evolving thoughts.net/2013/01/27/pattern-recognition-neither-deduction-nor-induction. Consultado 24/04/2018. 
Wilkins, J. S.; Ebach, M. C. 2014. The Nature of Classification: Relationships and Kinds in the Natural Science. 4th Edition. London: Palgrave-McMillan.

\section{Notas}

${ }^{1}$ Aquí estoy utilizando sin mayores desarrollos una clasificación de las ciencias en naturales, semióticas y humanas que llevo sosteniendo desde los años 80 .

${ }^{2}$ De forma semejante a como ocurre en el rectángulo de las oposiciones de los enunciados cuantificados, a saber, afirmativos/negativos y universales/particulares: A, E, I, O Las oposiciones horizontales A/E e I/O (contrariedad y subcontrariedad) son simples; las verticales A/I y E/O (subalternancias) también. En cambio, las diagonales A/O e I/E (contradictoriales) son dobles.

${ }^{3}$ En contraste con Descartes en su Discurso o Darwin en su Origen, que nos narran una historia personal, la historia de su vida, que los ha conducido hasta el inicio de su obra definitiva.

${ }^{4}$ Se citará el texto de Shannon de acuerdo con la traducción del autor que se halla en Álvarez 2018b, con la numeración entre corchetes: [número de página correspondiente al texto original mecanografiado de Shannon].

5 "Una tradición de investigación es un conjunto de supuestos generales acerca de los entidades y procesos que se dan en un campo de estudio, y acerca de los métodos adecuados para investigar los problemas y construir las teorías en ese campo" (Laudan 1977, p.81).

${ }^{6}$ En relación con la simplificación, además de la idealización y la minimización, suele considerarse el tema de la abstracción, aunque prefiero no entrar en él, porque para los efectos de esta argumentación no es necesario y supondría distinguir temas clásicos como oponer la abstracción de la forma (tradición aristotélica) de la abstracción precisiva (tradición empirista del s. XIV hasta la modernidad), alejándonos de lo principal.

${ }^{7} \mathrm{Cf}$. Álvarez 2018a para un tratamiento pormenorizado del tema.

${ }^{8}$ Desde principios de los 80 vengo sosteniendo que las relaciones semánticas son representativas e incorporativas y que, por ello, hay modelos que son representaciones satisfactorias e incorporaciones eficaces. El último desarrollo de esta tesis se halla en Álvarez 2018.

${ }^{9} \mathrm{P}=$ premisa; $\mathrm{RE}=$ regla de eliminación; $\mathrm{RI}=$ regla de introducción. 